\title{
Evaluation of non-parametric identification techniques in second order models plus dead time
}

\author{
Carlos Robles-Algarín ${ }^{1}$, Omar Rodríguez ${ }^{2}$, Adalberto Ospino ${ }^{3}$ \\ ${ }^{1,2}$ Facultad de Ingeniería, Universidad del Magdalena, Colombia \\ ${ }^{3}$ Facultad de Ingeniería, Universidad de la Costa, Colombia
}

\begin{tabular}{l} 
Article Info \\
\hline Article history: \\
Received Nov 30, 2019 \\
Revised Jun 1, 2020 \\
Accepted Jun 13, 2020 \\
\hline
\end{tabular}

\section{Keywords:}

Matlab PID tuner

Non-parametric techniques

System identification

Underdamped system

\begin{abstract}
In this paper, a set of non-parametric identification techniques are used in order to obtain second order models plus dead time for an underdamped system. Initially, non-parametric techniques were used to identify the system from the temperature data of a coal-heated oven. In this case, the identification techniques proposed by Stark, Jahanmiri-Fallahi and Ogata were used, which require obtaining two or three points of the step response for the system under study. In addition, the Matlab PID Tuner app was used to identify the underdamped system and compare the results with the other methods. The results show that the PID Tuner and the method proposed by Ogata are the ones that best represent the dynamics of the underdamped system, taking into account the values for the integral absolute error (IAE) and the correlation coefficient. With the Stark method an IAE of 181.56 was obtained, while with the PID Tuner the best performance was achieved with an IAE of 21.59. In terms of the results obtained with the cross correlation, the best performance was achieved with the PID tuner and the Stark method.
\end{abstract}

Copyright (C) 2020 Institute of Advanced Engineering and Science. All rights reserved.

Corresponding Author:

Carlos Robles-Algarín,

Facultad de Ingeniería,

Universidad del Magdalena,

Carrera 32 No. 22-08, Santa Marta, Magdalena, Colombia.

Email: croblesa@unimagdalena.edu.co

\section{INTRODUCTION}

One of the main blocks of a control system is represented by the controller, which is a device responsible for calculating the error deviation between a measured value and a reference value or set point. For the tuning of these controllers, an analytical process is carried out that allows obtaining the design parameters taking into account the variable to be controlled and the dynamics of the plant under study [1]. Before designing the controller, it is necessary to identify the system dynamics. This process requires using an excitation and storing the input and output data, in order to obtain a model that can correctly describe the system behavior [2]. In this context, identification techniques arise, which use statistical methods to design mathematical models from experimental data of a process [3, 4].

Depending on the model obtained, the identification techniques can be classified into parametric and non-parametric. In parametric techniques the model elements are calculated in order to minimize the error between the process under study and the model [5-7]. They are classified in time domain techniques and frequency domain techniques, which can be used to estimate parameters of continuous and discrete models [8].

Non-parametric techniques are characterized by representing the dynamics of the system with a nonfinite number of parameters [9-12]. They are classified into frequency techniques, correlation analysis and transient response analysis. In this work, non-parametric techniques based on the analysis of the transient response are used, which aim to obtain the system model in response to a step or impulse [13]. From a set of 
experimental data on the temperature of a coal-heated oven, the identification of a second-order system plus dead time was performed, using the structure of a transfer function for an underdamped system. To obtain the models, it was necessary to identify the gain, dead time, damping factor and the natural frequency of the system.

Specifically, the methods of Stark and Jahanmiri-Fallahi were used, which are ideal for the identification of second-order systems plus dead time and need three points on the output response [14]. In addition, the method proposed by Ogata was used to identify a second-order underdamped system plus dead time, based on the step response [15]. In parallel with the methods studied, the Matlab PID Tuner was used to identify the systems under study from a set of experimental data [16].

In the literature, there are investigations in which identification techniques have been used in overdamped and underdamped systems. In the work done in [17], a PID tuning methodology was proposed to obtain the mathematical model of high-order plants with oscillating dynamics. The researchers in [18] used different non-parametric identification methods applied in a linear pressure process. The results were evaluated in terms of performance indices such as modeling time and the IAE.

The authors in [19] used non-parametric techniques for modeling power curves of a wind turbine. The results obtained were contrasted with data from a wind farm in Canada. In the same field of application, the authors in [20] used non-parametric methods for interharmonic estimation in photovoltaic systems. Finally, in the work developed in [21] the modeling of linear systems with non-parametric methods was performed, for which periodic excitation signals were used.

In addition to the above, research has recently been carried out using soft computing techniques to perform non-parametric identification of dynamic systems. The authors in [22] made the identification of a liquid slosh plant using the Hammerstein model based on the Grey Wolf Optimizer method. The results obtained demonstrated that the proposed generic model has good potential to identify this type of process.

In [23] the authors proposed an improved version of the Sine Cosine Algorithm, in order to acquire and optimize a dynamic model for a twin rotor system, with which good performance and excellent precision in the process of identification was obtained. Furthermore, the researchers in [24] proposed a novel method for the identification of bilinear systems with Gaussian noises. Finally, in [25] the authors presented a comprehensive review of the computational techniques used in the literature to identify nonlinear systems.

Taking into account the literature review, this work proposes as a novelty the integration of methods that use two and three points of the step response curve to implement the identification process. The results obtained in terms of performance indices are highlighted. This paper is organized as follows: in section 2 the materials and methods are presented, in which the identification methods applied in one second order system plus dead time are detailed. In section 3, the results and the analysis of the models are presented, using reference parameters such as the IAE, correlation coefficient and cross correlation. Finally, conclusions and references are presented.

\section{RESEARCH METHOD}

\subsection{Underdamped system}

For this case, 309 samples of a variable of temperature from a coal-heated oven were used. Data were acquired in a prototype oven owned by the Magma Ingeniería research group at the Universidad del Magdalena, for which an Arduino Uno board and a PT-100 temperature sensor were used. The temperature values were stored in the Arduino board memory with a sampling time of $0.1 \mathrm{~s}$ and a resolution of 10 bits. These data are available in a file with a mat extension that was imported to Matlab using the load command. The temperature data was plotted to analyze the behavior in the time domain. Figure 1 shows that the data needs to be filtered in order to obtain the mathematical model of the process.

After a data analysis, repeated values in some time intervals were detected. In addition, it can be seen that the temperature is taking negative values. Due to the nature of the process, these values are not admissible and must be removed from the data vector. To eliminate the negative data, they were first replaced by 0 and then a linear interpolation was performed. The results are shown in Figure 2, in which it can be seen that there are no negative temperature data, but it is still necessary to apply a filtering stage before proceeding with the application of the identification methods.

For the filtering, a zero-phase filter [26] was implemented, which consists of making the average at each point with the surrounding points. If this average is made exactly over a period of the frequency to be eliminated, an average is being made over the period and therefore the contribution of this frequency with all its harmonics is eliminated. The appropriate Matlab function to apply this filter is the filtfilt function. With this function the filtering in the frequency space is carried out from the coefficients $a$ and $b$ of the transfer function $\mathrm{H}(\mathrm{w})$, as can be seen in (1) 


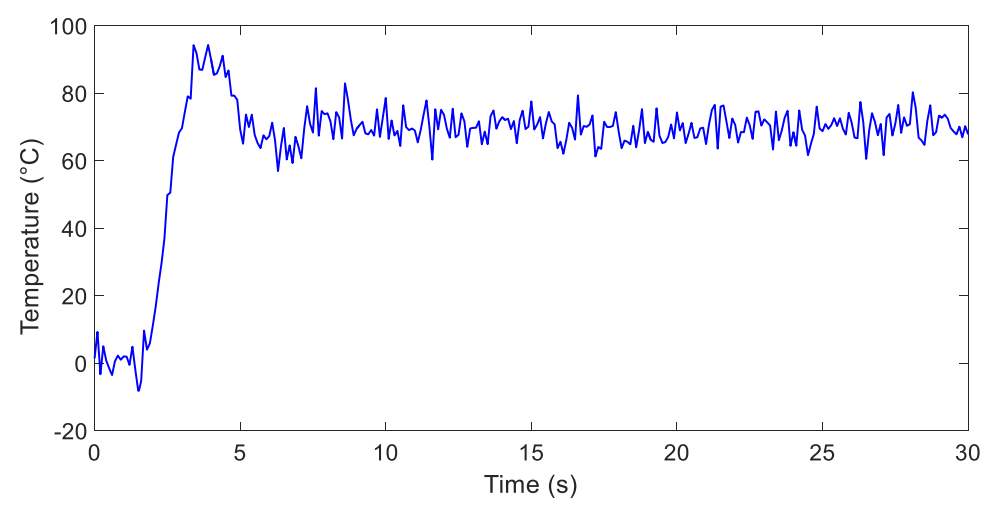

Figure 1. Experimental data of the underdamped system

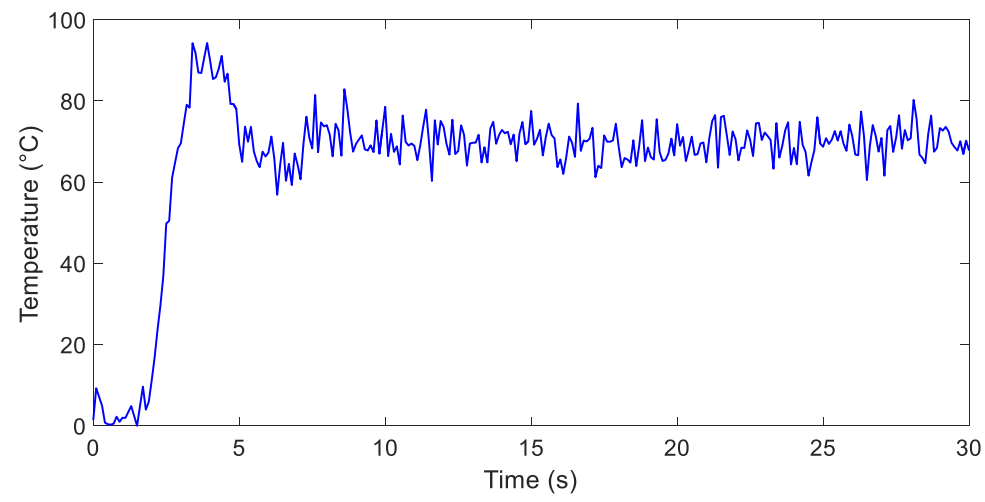

Figure 2. Experimental data without negative samples

$$
H(w)=\frac{b_{1} z^{0}+b_{2} z^{-1}+b_{3} z^{-3}+\cdots}{a_{1} z^{0}+a_{2} z^{-1}+a_{3} z^{-3}+\cdots}
$$

In the case of zero-phase filters, the numerator coefficients $\left(b_{1}, b_{2} \ldots\right)$ are all equal and equivalent to the inverse of the number of points on which the average is made, while the denominator is equal to $1(\mathrm{a}=1)$.

A good representation of the temperature data was obtained with the zero-phase filter, averaging with 10 points and eliminating the offset. The results can be as shown in Figure 3, which correspond to a second-order underdamped system plus dead time.

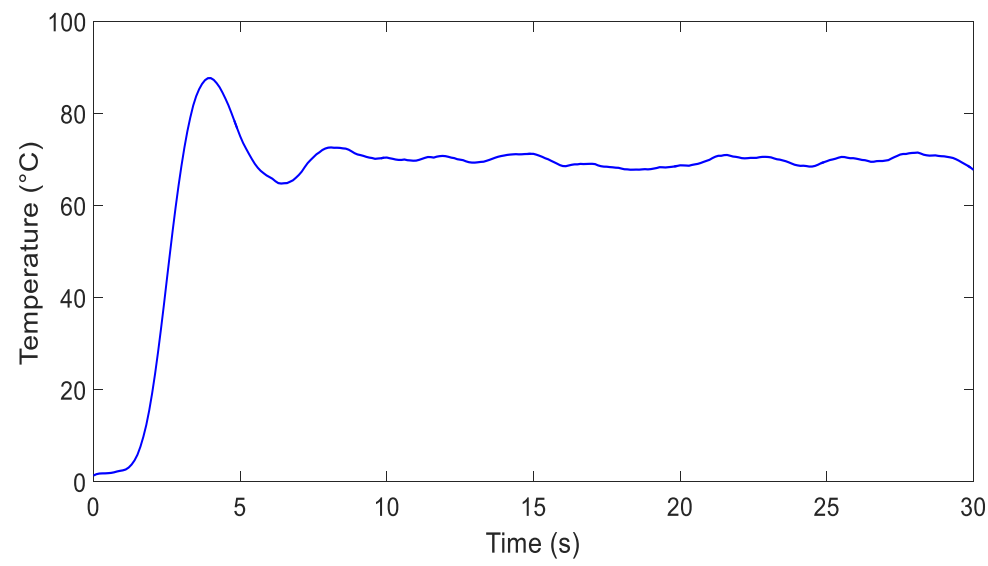

Figure 3. Temperature data filtered without offset 


\subsubsection{Identification with the stark method}

The first method used to identify the underdamped system of Figure 3 is that proposed by Stark [14]. In this method it is necessary to find the values in which the response of the system is $15 \%, 45 \%$ and $75 \%$ of the steady state value. To find the steady state value, the values where the signal tends to stabilize were averaged, approximately between $\mathrm{t}=10 \mathrm{~s}$ and $\mathrm{t}=30 \mathrm{~s}$, resulting in $68.3810{ }^{\circ} \mathrm{C}$. Taking this value as a reference, the times found are:

$$
\begin{aligned}
& t_{15 \%}=t_{68.3810 \times 0.15}=t_{10.2571}=1.75 \mathrm{~s} \\
& t_{45 \%}=t_{68.3810 \times 0.45}=t_{30.7714}=2.25 \mathrm{~s} \\
& t_{75 \%}=t_{68.3810 \times 0.75}=t_{51.2857}=2.65 \mathrm{~s}
\end{aligned}
$$

Once the three times were obtained, the parameter $\mathrm{x}$ was calculated from (5).

$$
x=\frac{t_{45 \%}-t_{15 \%}}{t_{75 \%}-t_{15 \%}}=\frac{2.25-1.75}{2.65-1.75}=0.5556
$$

Subsequently, the damping factor calculation was performed using (6).

$$
\zeta=\frac{0.0805-5.547(0.475-x)^{2}}{x-0.356}=0.2228
$$

Next, the calculation of function $\mathrm{f}_{2}$ was performed, taking into account the value of the damping factor.

$$
\begin{aligned}
& f_{2}(\zeta)= \begin{cases}2.6 \zeta-0.6 & \zeta \geq 1 \\
0.708(2.811)^{\zeta} & \zeta<1\end{cases} \\
& f_{2}(\zeta)=0.708(2.811)^{\zeta}=0.8913
\end{aligned}
$$

Then, the natural frequency was found with (8).

$$
\omega_{n}=\frac{1}{\tau}=\frac{f_{2}(\zeta)}{t_{75 \%}-t_{15 \%}}=0.9903
$$

With the value of the natural frequency, the function $\mathrm{f}_{3}$ was calculated and then the dead time $t_{0}$

$$
\begin{aligned}
& f_{3}(\zeta)=0.922(1.66)^{\zeta}=1.0322 \\
& t_{0}=t_{45 \%}-\frac{f_{3}(\zeta)}{\omega_{n}}=1.2077 \mathrm{~s}
\end{aligned}
$$

Finally, the calculation of the gain $\mathrm{k}_{\mathrm{p}}$ was performed.

$$
k_{P}=\frac{\Delta y}{\Delta u}=\frac{68.3810}{1}=68.3810
$$

With the calculated values of the natural frequency, the damping factor, the gain of the process and the dead time, the mathematical model of the process denoted as $\mathrm{G}_{\mathrm{s} 1}(\mathrm{~s})$ was obtained.

$$
\begin{aligned}
& G_{S 1}(s)=\frac{\omega_{n}{ }^{2} k_{p}}{s^{2}+2 \zeta \omega_{n} s+\omega_{n}{ }^{2}} e^{-t_{0} s} \\
& =\frac{67.06}{s^{2}+0.4413 s+0.9807} e^{-1.21 s}
\end{aligned}
$$

\subsubsection{Identification with the Jahanmiri-Fallahi method}

Like the Stark method, this method is based on obtaining three reference points from the step response of the model to be identified [27, 28]. The two options offered by this method were implemented. Initially the values $t_{2}, t_{70}$ and $t_{90}$ were used, which the times necessary for the output response are staying 
within 2,70 and $90 \%$ of the final value. The values of $t_{5}, t_{70}$ and $t_{90}$ were also used, where the only difference is the use of time $t_{5}$ in which the response staying within $5 \%$ of the final value.

For case 1 , the values of $t_{2}, t_{70}$ and $t_{90}$ were identified from Figure 3.

$$
t_{2 \%}=1.11 \mathrm{~s}, \quad t_{70 \%}=2.6 \mathrm{~s}, \quad t_{90 \%}=2.87 \mathrm{~s}
$$

Subsequently, the dead time was defined;

$$
t_{m}=t_{2 \%}=1.11 \mathrm{~s}
$$

The calculation of the reference constant was performed as shown in (15).

$$
\eta=\frac{t_{90}-t_{70}}{t_{90}-t_{m}}=\frac{2.87-2.6}{2.87-1.11}=0.1534
$$

Taking into account the value of the reference constant, the damping coefficient was calculated.

$$
\begin{aligned}
& \text { for } \eta \leq 0.4771 \rightarrow \zeta=\sqrt{\frac{0.4844651-0.75323499 \eta}{1-2.0946444 \eta}} \\
& \text { for } \eta \geq 0.4771 \rightarrow \zeta=13.9352 \\
& \text { for } \eta \leq 0.4771 \rightarrow \zeta=0.7373
\end{aligned}
$$

With the value of the damping coefficient, the calculation of the time constant was performed

$$
\tau=\frac{t_{90}-t_{m}}{0.424301+4.62533 \zeta-2.65412 e^{-\zeta}}=0.6862 \mathrm{~s}
$$

The gain $\mathrm{k}_{\mathrm{p}}$ is the same as calculated in the Stark method. With all the above values, the transfer function $\mathrm{G}_{\mathrm{JF} 1}(\mathrm{~s})$ was obtained.

$$
G_{J F 1}(s)=\frac{k_{p} e^{-t_{m} s}}{\tau^{2} s^{2}+2 \zeta \tau s+1}=\frac{68.38 e^{-1.11 s}}{0.4709 s^{2}+1.012 s+1}
$$

For case 2, the values of $t_{5}, t_{70}$ and $t_{90}$ were used from Figure 3 . For $t_{m}=t_{5}=1.41 \mathrm{~s}$, the calculation of the reference constant, damping coefficient and time constant was performed. This way, the parameters shown in (20) were obtained.

$$
\eta=0.1849, \zeta=0.7506, \tau=0.6659
$$

Then, the transfer function $\mathrm{G}_{\mathrm{JF} 2}(\mathrm{~s})$ was obtained.

$$
G_{J F 2}(s)=\frac{k_{p} e^{-t_{m} s}}{\tau^{2} s^{2}+2 \zeta \tau s+1}=\frac{68.38 e^{-1.41 s}}{0.4434 s^{2}+0.9996 s+1}
$$

\subsubsection{Identification with the Ogata method}

In this method all design parameters are extracted taking into account the analysis of the transient response of a second order system plus dead time [15]. Initially, the calculation of the maximum overshoot of Figure 3 was performed.

$$
M_{p}=\frac{y\left(t_{p}\right)-y(s s)}{y(s s)}=\frac{86.3-68.38}{68.38}=0.2621
$$

$y\left(t_{p}\right)$ equals the value of the output at peak time $t_{p}$. In the case of the system under study, the peak time is $\mathrm{t}_{\mathrm{p}}=4 \mathrm{~s}$ and the output value at that time is $\mathrm{y}\left(\mathrm{t}_{\mathrm{p}}\right)=86.3$.

$\mathrm{y}(\mathrm{ss})$ is the stable state value, which is 68.38 . With the value of $\mathrm{M}_{\mathrm{p}}$, the damping factor can be obtained with (23). 


$$
\zeta=\sqrt{\frac{1}{1+\left(\frac{\pi}{\ln \left(M_{p}\right)}\right)^{2}}}=0.3921
$$

With the peak time $t_{p}=4 \mathrm{~s}$, the natural frequency $\mathrm{w}_{\mathrm{n}}$ is found with (24).

$$
w_{d}=\frac{\pi}{t_{p}}=0.7854, \quad w_{n}=\frac{w_{d}}{\sqrt{1-\zeta^{2}}}=0.8538
$$

The process gain $\left(\mathrm{k}_{\mathrm{p}}\right)$ is the value of 68.38. In addition, it was determined from Figure 3 that the dead time is $t_{0}=1.1 \mathrm{~s}$. With the parameters calculated above, the process transfer function $\mathrm{G}_{\mathrm{T} 1}(\mathrm{~s})$ is obtained.

$$
G_{T 1}=\frac{\omega_{n}{ }^{2} k_{P}}{s^{2}+2 \zeta \omega_{n} s+\omega_{n}^{2}} e^{-t_{0} s}=\frac{49.85}{s^{2}+0.6695 s+0.729} e^{-1.1 s}
$$

\subsubsection{Identification with the Matlab PID Tuner app}

Using the Matlab PID Tuner app [16], the system identification was performed from the original (unfiltered) data that is described in Figure 1. For this, the plant Identification tool was used and a second order underdamped model with delay was selected (Underdamped Pair). It is noteworthy that the PID tuner uses a proprietary tuning algorithm developed by MathWorks. In addition, the Auto Estimate tool was used to improve the results obtained in the model. The results are shown in Figure 4. Equation (26) shows the $\mathrm{G}_{\text {Tuner }}$ transfer function with the parameters $\mathrm{K}=69.957, \mathrm{~T}_{\mathrm{w}}=0.68764, \zeta=0.357$ and $\tau=1.5127$ obtained with the PID Tuner.

$$
G_{\text {Tuner }}=\frac{K}{T_{w}^{2} s^{2}+2 \zeta T_{w} s+1} e^{-\tau s}=\frac{69.957}{0.4728 s^{2}+0.491 s+1} e^{-1.5127 s}
$$

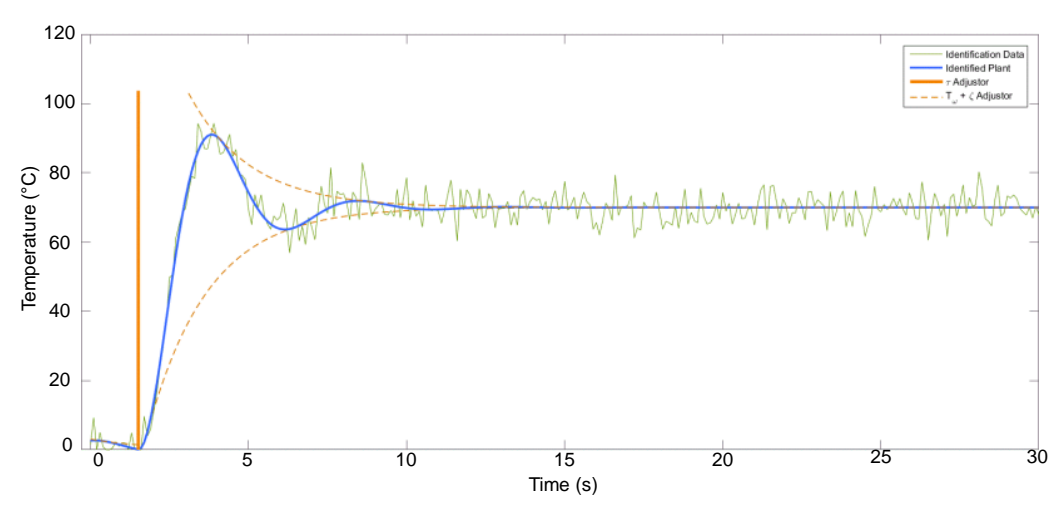

Figure 4. Identification with the Matlab PID Tuner

\subsection{Model validation}

In order to determine the accuracy in the identification process, the IAE was calculated for all methods [29]. See (27),

$$
I A E=\int_{0}^{\alpha}\left|y_{p}(t)-y_{m}(t)\right| d t
$$

where $y_{p}(t)$ is the plant output, which corresponds to the temperature in degree Celsius of the experimental data. $y_{\mathrm{m}}(\mathrm{t})$ is the identified model for the temperature in degree Celsius.

This index represents the area between the plant response and the identified model response. If the IAE $\rightarrow 0$ then $\mathrm{y}_{\mathrm{m}}(\mathrm{t}) \rightarrow \mathrm{y}_{\mathrm{p}}(\mathrm{t})$. Thus, as this performance index decreases, the accuracy of the model will be better. In Matlab the trapz command was used, which allows the calculation of the approximate integral of a data vector, using the trapezoidal method. 
Additionally, in order to measure the similarity between the signals, the calculation of the Pearson correlation coefficient ( $r$ ) and the cross correlation (R) was performed [30]. For this purpose, the Matlab xcorr and corrcoef commands were used.

The results obtained with the correlation coefficient were interpreted according to the following cases:

- If $0<\mathrm{r}<1$, indicates that there is a positive correlation.

- If $\mathrm{r}=1$, it is a perfect positive correlation, which means that if one variable increases the other increases in the same proportion.

- If $-1<\mathrm{r}<0$, indicates that there is a negative correlation, which means an inverse relationship between the variables.

\section{RESULTS AND DISCUSSION}

Figure 5 shows the step response for each of the transfer functions obtained with the 5 identification methods implemented in this work. It can be seen that the signals obtained with the PID Tuner app and with the methods of Stark and Ogata are the ones that best represent the dynamics of the original signal. In order to perform a statistical analysis of the results, the calculations of the IAE and the correlation coefficient were performed, which are shown in Table 1. It can be seen that the best IAE was obtained with the PID Tuner and the Ogata method with values of 21.59 and 62.07 respectively. However, the values calculated in the 5 methods reflect that the transfer functions show differences with respect to the temperature data of the original process. On the other hand, correlation coefficients indicating a positive correlation were obtained with all the methods. The best result, according to this coefficient, was obtained with the PID Tuner app with a value of 0.9787 as shown in Table 1. Finally, Figure 6 shows the cross correlation for each of the methods in relation to the original signal. In this case, the best results were obtained with the PID Tuner and the Stark method.

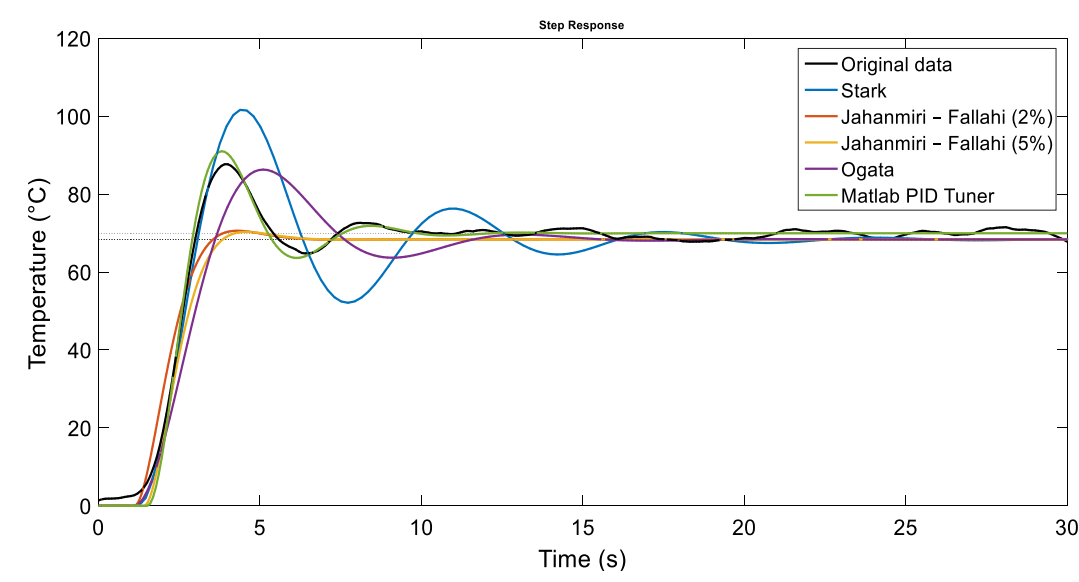

Figure 5. Step response for the underdamped system with the 5 methods

Table 1. IAE and correlation coefficient for the underdamped system

\begin{tabular}{cccc}
\hline Method & Transfer Function & IAE & r \\
\hline Stark & $G_{S 1}=\frac{67.06}{s^{2}+0.4413 s+0.9807} e^{-1.21 s}$ & 181.56 & 0.9367 \\
Jahanmiri-Fallahi (t2\%) & $G_{J F 1}(s)=\frac{68.38 e^{-1.11 s}}{0.4709 s^{2}+1.012 s+1}$ & 133.28 & 0.9761 \\
Jahanmiri-Fallahi (t5\%) & $G_{J F 2}(s)=\frac{68.38 e^{-1.41 s}}{0.4434 s^{2}+0.9996 s+1}$ & 329.94 & 0.9747 \\
Ogata & $G_{T 1}=\frac{49.85}{s^{2}+0.6695 s+0.729} e^{-1.1 s}$ & 62.07 & 0.9505 \\
Matlab PID Tuner & $G_{\text {Tuner }}=\frac{6.4728 s^{2}+0.491 s+1}{0.1 .51 s}$ & 21.59 & 0.9787 \\
\hline
\end{tabular}




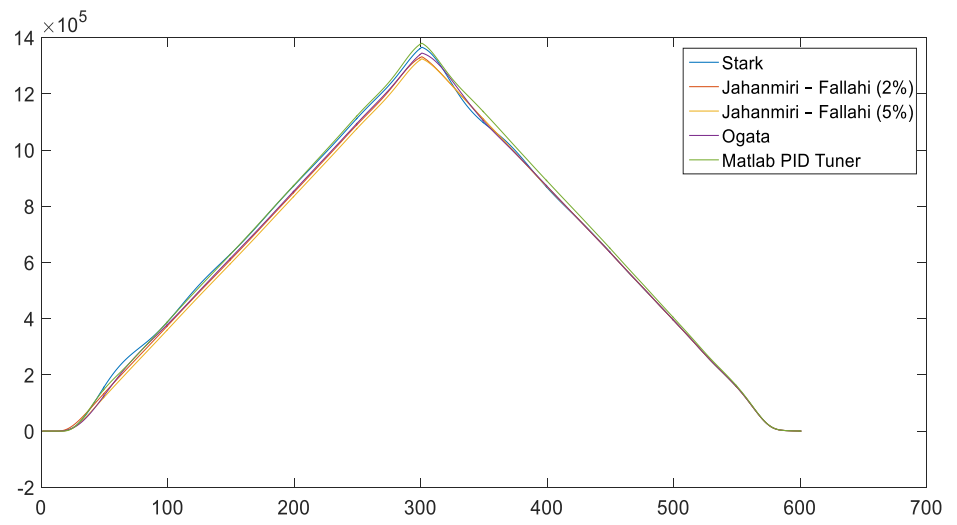

Figure 6. Results obtained with cross correlation with the methods under study

\section{CONCLUSION}

It can be concluded that non-parametric techniques can be applied for the identification of second order underdamped systems plus dead time. It was established that the PID Tuner tool and the method proposed by Ogata are the ones that best represent the transient response of the plant under study. Also, it was possible to demonstrate the need to evaluate other identification techniques reported in the literature such as neural networks, in order to improve the results obtained in relation to the integral absolute error. On the other hand, the calculation of Pearson's correlation coefficient made it possible to verify that there is a positive correlation between the methods applied and the experimental data.

\section{REFERENCES}

[1] C. Lorenzini, et al., "The generalized forced oscillation method for tuning PID controllers," ISA Transactions, vol. 87 , pp. $68-87,2019$.

[2] W. Ariza Ramirez, et al., "Non-parametric dynamic system identification of ships using multi-output Gaussian Processes," Ocean Engineering, vol. 166, pp. 26-36, 2018

[3] S. Abrashov, et al., "Optimal input design for continuous-time system identification," Communications in Nonlinear Science and Numerical Simulation, vol. 60, pp. 92-29, 2018.

[4] E. O. Kontis, et al., "Modal analysis of active distribution networks using system identification techniques," International Journal of Electrical Power and Energy Systems, vol. 100, pp. 365-378, 2018.

[5] C. S. Lin, "Frequency-domain approach for the parametric identification of structures with modal interference," Journal of Mechanical Science and Technology, vol. 33, pp. 4081-4091, 2019.

[6] P. Frank Pai, "Time-frequency analysis for parametric and non-parametric identification of nonlinear dynamical systems," Mechanical Systems and Signal Processing, vol. 36, pp. 332-353, 2013.

[7] I. Uyanık, et al., "Parametric Identification of Hybrid Linear-Time-Periodic Systems," IFAC-PapersOnLine, vol. 49, pp. 7-12, 2016.

[8] K. Dziedziech, et al., "Combined non-parametric and parametric approach for identification of time-variant systems," Mechanical Systems and Signal Processing, vol. 103, pp. 295-311, 2018.

[9] R. Voorhoeve, et al., "Non-parametric identification of multivariable systems: A local rational modeling approach with application to a vibration isolation benchmark," Mechanical Systems and Signal Processing, vol. 105, pp. 129-152, 2018.

[10] T. C. Yuan, et al., "Nonparametric identification of nonlinear piezoelectric mechanical systems," Journal of Applied Mechanics, Transactions ASME, vol. 85, pp. 1-13, 2018.

[11] A. Klepka and T. Uhl, "Identification of modal parameters of non-stationary systems with the use of wavelet based adaptive filtering," Mechanical Systems and Signal Processing, vol. 47, pp. 21-34, 2014.

[12] Y. Muñoz, et al., "Implementation of a frequency control in a biomass gasifier system," International Journal of Electrical and Computer Engineering (IJECE), vol. 9, no. 1, pp. 66-77, 2019.

[13] V. Volkova, "Development of methods for nonparametric identification of models of mechanical systems," 11th International Scientific Conference on Modern Building Materials, Structures and Techniques, vol. 57, pp. 1230-1235, 2013.

[14] V. Alfaro, "Identificación de procesos sobreamortiguados utilizando técnicas de lazo abierto," Ingeniería, vol. 1, pp. 11-25, 2001.

[15] K. Ogata, "Modern Control Engineering," London: Pearson, 2010.

[16] "pidTuner," Matworks, 2019. [Online]. Available: https://goo.gl/h1YMQF

[17] D. F. Novella-Rodríguez, et al., "Delayed Model Approximation and Control Design for Under-Damped Systems," IFAC-PapersOnLine, vol. 50, pp. 1316-1321, 2017. 
[18] R. Priyadharshini and S. Rakeshkumar, "Comparative analysis of auto-tuners for pressure tank process," International Journal of Applied Engineering Research, vol. 9, pp. 5291-5298, 2014.

[19] M. Mehrjoo, et al., "Wind turbine power curve modeling for reliable power prediction using monotonic regression," Renewable Energy, vol. 147, pp. 214-222, 2020.

[20] V. Ravindran, et al., "Comparison of a non-parametric and parametric method for interharmonic estimation in PV systems," IEEE Milan PowerTech, 2019.

[21] J. Schoukens, et al., "Nonparametric Data-Driven Modeling of Linear Systems: Estimating the Frequency Response and Impulse Response Function," IEEE Control Systems, vol. 38, pp. 49-88, Aug. 2018.

[22] M. A. Ahmad, et al., "Grey wolf optimizer for identification of liquid slosh behavior using continuous-time hammerstein model," Bulletin of Electrical Engineering and Informatics (BEEI), vol. 9, no. 2, pp. 542-549, 2020.

[23] S. Mohammad, et al., "Elimination-Dispersal Sine Cosine Algorithm for a Dynamic Modelling of a Twin Rotor System," Lecture Notes in Electrical Engineering, vol. 632, pp. 167-178, 2020.

[24] Z. Wang, et al., "Levy-particle swarm optimization intelligent search-based iterative identification for nonparametric models of bilinear systems with Gaussian mixture noises," Transactions of the Institute of Measurement and Control, vol. 41, pp. 3970-3978, 2019.

[25] G. Quaranta, et al., "A review on computational intelligence for identification of nonlinear dynamical systems," Nonlinear Dyn, vol. 99, pp. 1709-1761, 2020.

[26] Wang, et al., "Parallel-type fractional zero-phase filtering for ECG signal denoising," Biomedical Signal Processing and Control, vol. 18, pp. 36-41, 2015

[27] A. Jahanmiri and H. Fallahi, "New methods for process identification and design of feedback controller," Chemical Engineering Research and Design, vol. 75, pp. 519-522, 1997.

[28] C. T. Chen and S. T. Chen, "Design of a Sliding Mode Control System Based on an Identified SPODT Model," IFAC Proceedings Volumes, vol. 37, no. 1, pp. 827-832, 2004.

[29] J. Liu, et al., "IAE performance based signal complexity measure," Measurement: Journal of the International Measurement Confederation, vol. 75, pp. 255-262, 2015.

[30] Y. Zhang, et al., "Pearson correlation coefficient of current derivatives based pilot protection scheme for longdistance LCC-HVDC transmission lines," International Journal of Electrical Power and Energy Systems, vol. 116, 2020.

\section{BIOGRAPHIES OF AUTHORS}

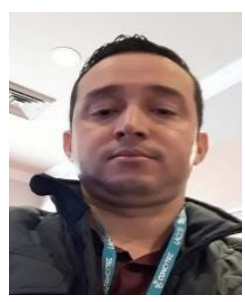

Carlos Robles Algarín, associate professor in the Department of Engineering at the Universidad del Magdalena, with an academic and research experience of 13 years. He is an Electronic Engineer, Master in Control Engineering and Process Automation and Ph.D. in Science and Technology. His areas of research interest are control systems, design of electronic systems and photovoltaic solar energy.

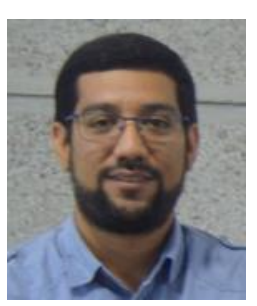

Adalberto Ospino Castro, he received his master degree in Control Engineering and Process Automation and the Ph.D. degree in Science and Technology in the University Rafael Belloso Chacín (URBE) and Degree Electronics Engineer from the Universidad Tecnológica de Bolívar (UTB). Lecturer and researcher Department in Energy in the Universidad de la Costa (CUC), responsible for the research area in renewable energy.

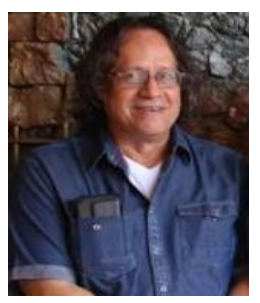

Omar Rodríguez Álvarez, associate professor in the Department of Engineering at the Universidad del Magdalena, with an academic and research experience of 25 years. He is an Electronic Engineer and Master in Education. His areas of interest are dynamic systems and linear and non-linear control systems. 